\title{
Important bee plants for stingless bees (Melipona and Trigonini) and Africanized honeybees (Apis mellifera) in neotropical habitats: a review
}

\author{
M Ramalho, A Kleinert-Giovannini, VL Imperatriz-Fonseca \\ Departamento de Ecologia Geral do Instituto de Biocièncias, Universidade de São Paulo, 05508, \\ São Paulo, Brazil
}

(Received 13 July; accepted 3 August 1990)

\begin{abstract}
Summary - This paper reports on pollen and nectar sources for stingless bees in neotropical habitats. Some 288 species were considered important bee plants. Eighty of these were important for the Africanized honeybee. Plant families with the largest number of important species for both bee groups are also among those with the greatest diversity in neotropical regions. Some differences among the bee groups are highlighted
\end{abstract}

Africanized honeybee / Melipona / Trigonini / bee plant / neotropics

\section{INTRODUCTION}

Studies of flower visiting by species of stingless bees and Africanized honeybees in neotropical regions of the Americas are still few and far between, and limited in scope, with variable objectives and methodologies (Absy and Kerr, 1977; Roubik, 1978, 1979; Iwama and Melhem, 1979; Absy et al, 1980, 1984; Engel and Dingemans-Bakels, 1980; Sommeijer et al, 1983; Imperatriz-Fonseca et al, 1984, 1987, 1989; Silveira et al, 1984; Ramalho et al, 1985, 1989; Roubik et al, 1986; Kleinert-Giovannini and Imperatriz-Fonseca, 1987; Kleinert-Giovannini et al, 1987; Cortopassi-Laurino and Ramalho, 1988; Ramalho, 1990). Thus any review such as this must be selective and preliminary in nature. Bee plants have been characterized most frequently in relation to observations of Africanized and European honeybees when visiting flowers, and this has yielded significant information on bee plants (Crane et al, 1984), but at the same time this makes comparison with other bee species difficult. As to studies of the floral origin of honey using pollen grains analysis (eg Barth, $1970 \mathrm{a}, \mathrm{b}$ ), they present estimates of nectar collecting from each plant species, but these are subject to errors of interpretation due to variations between different plant species in terms of pollen grains production. With a few exceptions, studies of bee communities (Sakagami et al, 1967; Sakagami and Laroca, 1971; Heithaus, 1979; Roubik, 1978, 1979; Cortopassi-Laurino, 1982; Laroca et al, 1982; Hakim, 1983; Opler, 1983; Orth, 1983; Camargo and Masukato, 1984; Knoll, 1985; Bortoli, 1987; Knoll et al, 1987; Almeida and Laroca, 1988; Knoll, 1990) fail to present data on the use of specific floral sources, since they deal with ecological questions at a different level of analysis, such as: diversity and phenology of bee species, characterization of syndromes and guilds of flower visitors, etc. 
Hence in this review of bee plant species in neotropical zones, we have only considered quantitative information on flower visiting which permits a comparison of the value of specific floral sources for stingless bees and for Africanized honeybees. Most of the data used were based on melissopalynological studies, whose positive and negative aspects are notorious (Louveaux et al, 1978).

\section{METHODS}

The important floral sources of pollen and nectar for stingless bees (Melipona and Trigonini) and Africanized honeybees in neotropical habitats were reviewed and a synthesis prepared. The species important for Africanized honeybee were included in the results taken from studies comparing Africanized honeybee and stingless bees. With regard to the plant species already included in the list according to the above criterion, supplementary information taken from studies of Africanized honeybees was added.

Most the data were obtained via a pollen analysis of the food collected by bees. In these cases, we selected only plant species with a pollen representativity exceeding $10 \%$, or from the pollen categories "frequent" (5-30\%), "abundant" $(>30 \%)$, "secondary" (15-45\%), and "predominant" (> 45\%), in accordance with classification schemes used by various authors (Iwama and Melhem, 1979; Engel and Dingemans-Bakels, 1980; Sommeijer et al, 1983; Imperatriz-Fonseca et al, 1984, 1987, 1989; Ramalho et al, 1985, 1989; Kleinert-Giovannini and Imperatriz-Fonseca, 1987; Ramalho, 1987; Cortopassi-Laurino and Ramalho, 1988). Absy's studies (Absy and Kerr, 1977; Absy et al, 1980, 1984) provided us with the plant species most frequently found in pollen and nectar samples, those most persistent throughout the year (in number of months), or those visited by the largest number of stingless bees. Roubik et al's study (1986) provided us with references to the plant species "heavily" utilized by bees for pollen. A few field studies were used to list plant species visited by $>5 \%$ of the total number of individuals of some species of stingless bee and Africanized honeybee on flowers (Sakagami and Laroca, 1971; Orth, 1983; Knoll, 1985; Knoll and Imperatriz-Fonseca, 1987; Almeida and Laroca, 1988; Knoll, 1990).

\section{RESULTS AND DISCUSSION}

The data on flower visiting by species of stingless bees and Africanized honeybee (Apis mellifera L) in neotropical habitats are summarized in table $\mathrm{I}$. Of the 23 studies mentioned, 5 compare Africanized honeybee and stingless bees, and a further 6 compare Melipona and Trigonini. In 6 of the studies, there is information on Melipona alone, and in 9 others, the information concerns only Trigonini. The data shown in table II are therefore biased as regards the number of plant species visited by the different groups of bees, or by 2 or more groups. The studies were conducted in areas with different types of vegetation, influenced in some cases by the presence of agriculture, pasture and or urbanization. Some studies were carried out in natural forests, a very few in grasslands or savannah. Herbaceous species and shrubbery, both ruderal and opportunistic, albeit originally under forest cover, are evidently abundant in areas with open vegetation.

Table II shows all the plant species with the highest levels of representativity in the diet of these bees in accordance with the quantitative studies listed in table I. Particularly in the case of the plant families of greatest significance for bees in neotropical zones, some common trends in latitudinal distribution may be detected. Generally speaking, the families with a larger number of species, ie Anacardiaceae, Compositae, Euphorbiaceae, Labiatae, Leguminosae, Melastomataceae, Moraceae, Myrtaceae, Palmae, Rubiaceae and Solanaceae, are also the most consistent pollen and nectar 


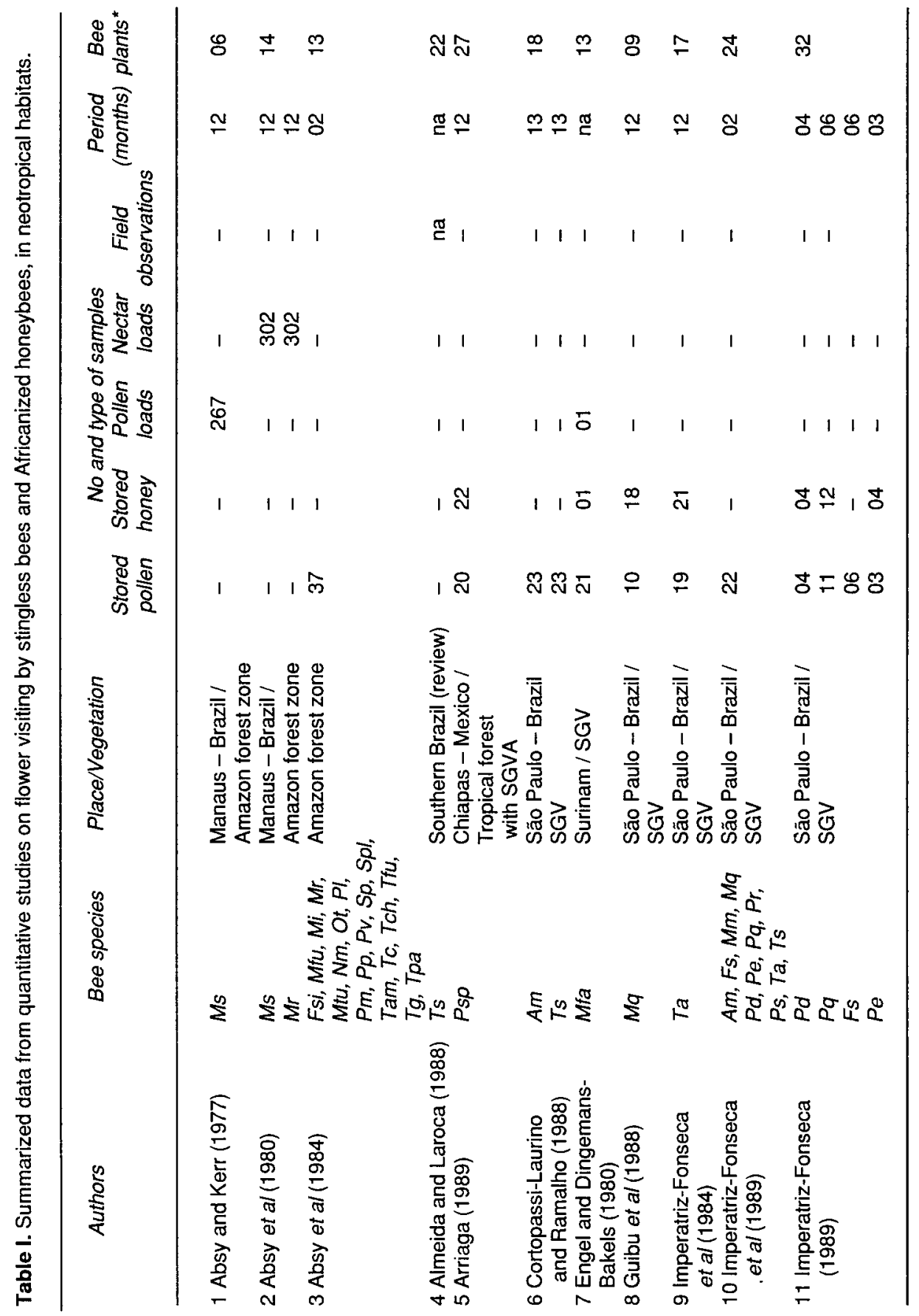




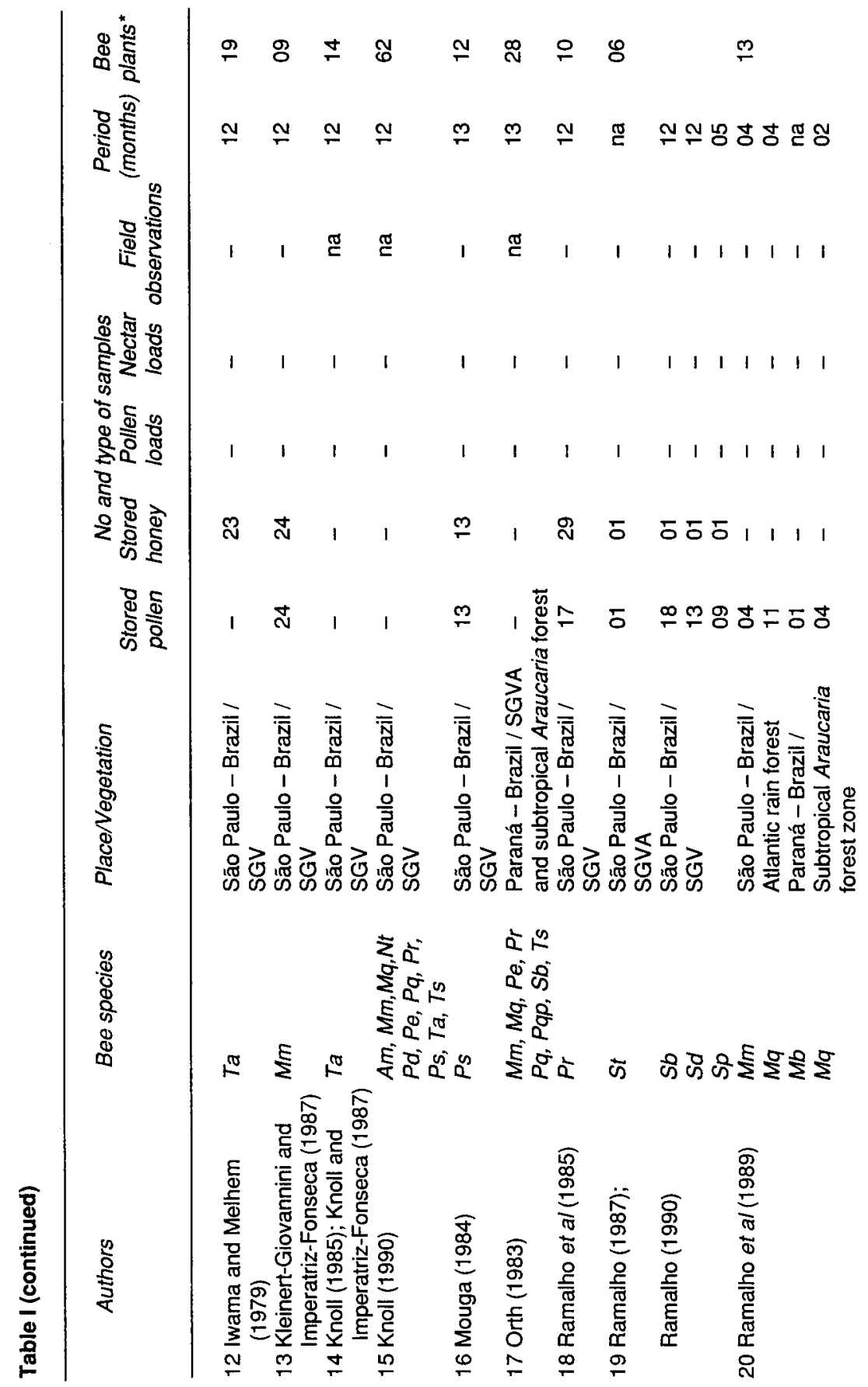




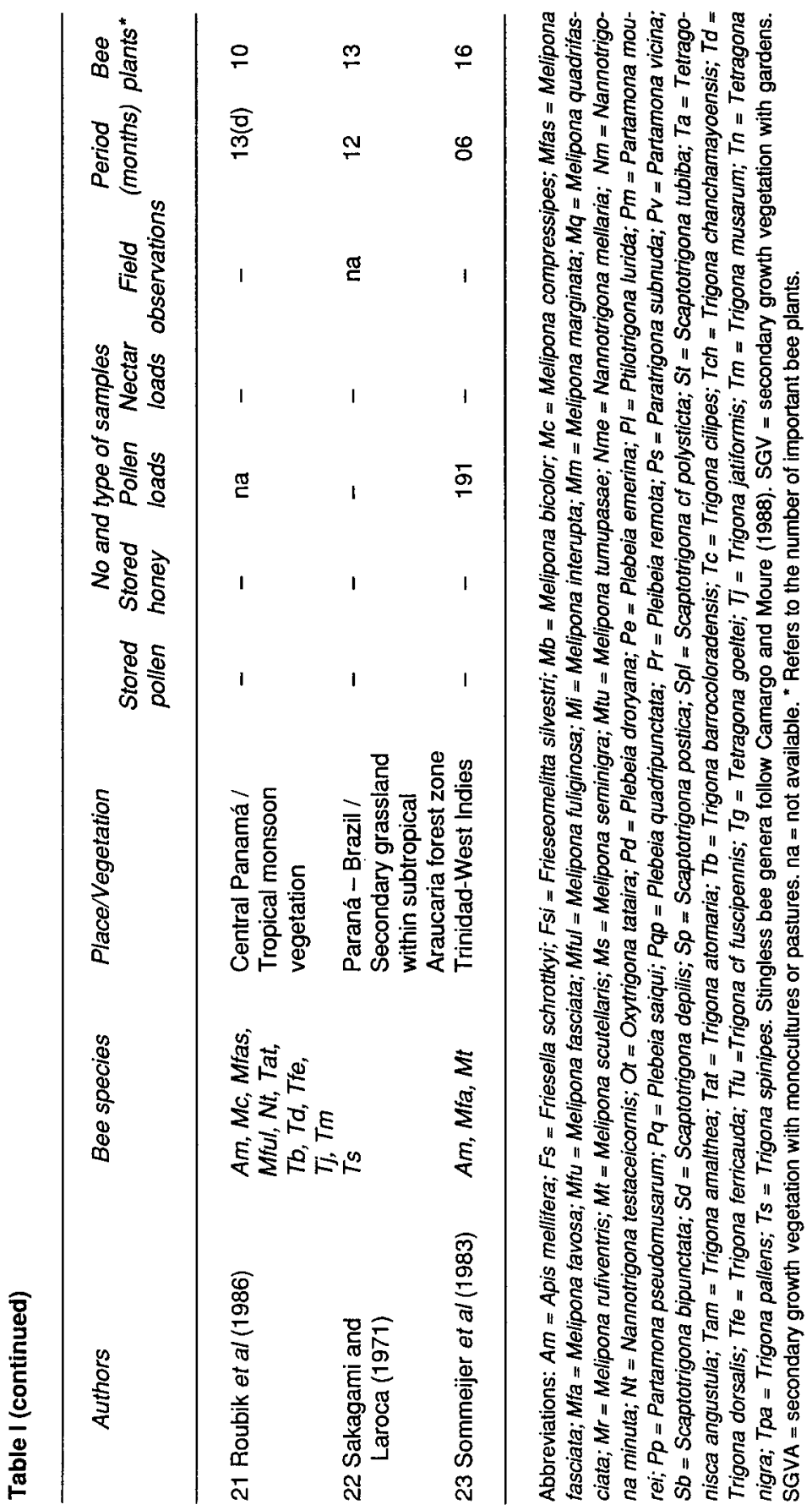


Table II. Important bee plants for stingless bees (Melipona and Trigonini) and Africanized honeybees (Apis mellifera) in neotropical habitats. Reference numbers are indicated in table I. (See $\mathrm{Me}$ thods for explanation on selection of important bee plants). $\mathrm{P}=$ pollen; $\mathrm{N}=$ nectar. + and $\mathrm{x}=$ species or genus, respectively, listed in the Directory of Important World Honey Sources (Crane et al, 1984). Data from studies with Africanized Apis mellifera (Santos, 1974; Roubik et al, 1984; Barth, 1989; Ramalho et al, 1990).

\begin{tabular}{|c|c|c|c|c|c|c|}
\hline Bee plants & Ref & $\begin{array}{l}\text { Stingle } \\
\text { Meli- } \\
\text { pona }\end{array}$ & $\begin{array}{c}\text { Ss bees } \\
\text { Trigo- } \\
\text { nini }\end{array}$ & $\begin{array}{c}\text { Africanized } \\
\text { Honeybees } \\
\text { A mellifera }\end{array}$ & $P$ & $N$ \\
\hline \multicolumn{7}{|l|}{ Acanthaceae } \\
\hline Eranthemum sp & 15 & & & $x$ & & \\
\hline $\begin{array}{l}\text { Thunbergia grandiflora } \\
\text { Agavaceae }\end{array}$ & +15 & & $x$ & & & \\
\hline Agave sisalana & * 15 & & $x$ & $x$ & & \\
\hline \multicolumn{7}{|l|}{ Aizoaceae } \\
\hline $\begin{array}{l}\text { Mesembryanthemum spectabile } \\
\text { Amaranthaceae }\end{array}$ & \multicolumn{5}{|c|}{ Amaranthaceae } & \\
\hline Alternanthera sp & 2 & $x$ & & & & $x$ \\
\hline Iresine celosia & 5 & & $\mathrm{x}$ & & & $x$ \\
\hline \multicolumn{7}{|l|}{ Anacardiaceae } \\
\hline Lithraea molleoides & 1015 & & $x$ & & $x$ & \\
\hline Mangifera indica & +16 & & $x$ & & $x$ & \\
\hline Schinus terebinthifolius & +81011 & & $\mathrm{x}$ & $\mathbf{\square}$ & & $x$ \\
\hline Spondias $\mathrm{sp}$ & $* 5$ & & $x$ & $\mathbf{\square}$ & $x$ & $x$ \\
\hline Spondias mombim & +3523 & $x$ & $\mathrm{x}$ & $\mathrm{x}$ & $\mathrm{x}$ & $\mathrm{x}$ \\
\hline Spondias raldkoferri & $\star 21$ & & $x$ & $x$ & $x$ & \\
\hline Tapirira guianensis & 23 & $x$ & $x$ & & $x$ & $x$ \\
\hline \multicolumn{3}{|l|}{ Araliaceae } & $x$ & & & \\
\hline Didymopanax morototomi & 7 & $x$ & & & $x$ & \\
\hline $\begin{array}{l}\text { Tetrapanax papirifera } \\
\text { Avicenniaceae }\end{array}$ & 911 & & $x$ & & $x$ & $x$ \\
\hline \multirow{2}{*}{\multicolumn{7}{|c|}{ Balsaminaceae }} \\
\hline & & & & & & \\
\hline Impatiens balsamina & 111518 & & $x$ & $\mathrm{x}$ & $x$ & \\
\hline I sultanii & 101115 & & $\mathrm{x}$ & $x$ & $x$ & $\mathrm{x}$ \\
\hline I walleriana & 5 & & $x$ & & $x$ & $x$ \\
\hline \multicolumn{7}{|l|}{ Bignoniaceae } \\
\hline Jacaranda oxyphyla & 4 & & $x$ & & & \\
\hline Parmentiera aculeata & 5 & & $x$ & & $x$ & \\
\hline Spathodea campanulata & 9 & & $x$ & & $x$ & \\
\hline $\begin{array}{l}\text { Tabebuia umbellata } \\
\text { Bixaceae }\end{array}$ & 11 & & $x$ & & $x$ & \\
\hline $\begin{array}{l}\text { Bixa orellana } \\
\text { Bombacaceae }\end{array}$ & 1 & $x$ & & & $x$ & \\
\hline Chorisia speciosa & 11 & & $x$ & & $x$ & \\
\hline
\end{tabular}


Table II (continued)

\begin{tabular}{|c|c|c|c|c|c|c|}
\hline Bee plants & Ref & $\begin{array}{c}\text { Stingle } \\
\text { Meli- } \\
\text { pona }\end{array}$ & $\begin{array}{c}\text { S bees } \\
\text { Trigo- } \\
\text { nini }\end{array}$ & $\begin{array}{c}\text { Africanized } \\
\text { Honeybees } \\
\text { A mellifera }\end{array}$ & $P$ & $N$ \\
\hline \multicolumn{7}{|l|}{ Boraginaceae } \\
\hline $\begin{array}{l}\text { Cordia alliodora } \\
\text { Burseraceae }\end{array}$ & +5 & & $x$ & & $x$ & $x$ \\
\hline Protium heptaphyllum & 123 & $x$ & $x$ & & $x$ & $x$ \\
\hline $\begin{array}{l}P \text { tenuifolium } \\
\text { Cannaceae }\end{array}$ & 21 & & $x$ & $x$ & $x$ & \\
\hline $\begin{array}{l}\text { Canna indica } \\
\text { Caprifoliaceae }\end{array}$ & 17 & & $x$ & & & \\
\hline $\begin{array}{l}\text { Sambucus australis } \\
\text { Caricaceae }\end{array}$ & 91119 & & $x$ & & $x$ & \\
\hline $\begin{array}{l}\text { Carica papaya } \\
\text { Caryophyllaceae }\end{array}$ & +5 & & $x$ & & $x$ & $x$ \\
\hline $\begin{array}{l}\text { Silene armeria } \\
\text { Celastraceae }\end{array}$ & 1415 & $x$ & $x$ & & & \\
\hline $\begin{array}{l}\text { Maytenus evonimoides } \\
\text { Clethraceae }\end{array}$ & 10 & & $x$ & & $x$ & \\
\hline $\begin{array}{l}\text { Clethrasp } \\
\text { Compositae }\end{array}$ & $* 5$ & & $x$ & & $x$ & $x$ \\
\hline Ageratum houstonianum & 5 & & $x$ & & & $x$ \\
\hline Ambrosia type & 9 & & $x$ & & & \\
\hline Aster laevis & 15 & & & $x$ & & \\
\hline Baccharis sp & ${ }^{*} 41521$ & & $x$ & $x$ & & \\
\hline Baccharis si & 619 & & $\mathrm{x}$ & $x$ & $x$ & \\
\hline B elaeagnoides & 17 & & $x$ & & & \\
\hline B erioclada & 17 & $x$ & $x$ & & & \\
\hline$B$ helicrysoides & 17 & & $x$ & & & \\
\hline$B$ meridionales & 17 & $x$ & $x$ & & & \\
\hline B oxyodonta & 17 & & $x$ & & & \\
\hline$B$ semiserrata & 17 & $x$ & $x$ & & & \\
\hline B spicata & 422 & & $x$ & & & \\
\hline Bidens sp & $\star \overline{12}$ & & $x$ & $\mathbf{a}$ & & $\mathrm{x}$ \\
\hline B pilosa & +1115 & & $x$ & $\bar{x}$ & $x$ & \\
\hline Chrysanthemum type & 11 & & $x$ & & $x$ & \\
\hline Chysanthemum maximum & 15 & & $x$ & & & \\
\hline Cirsium vulgare & 18 & $x$ & $x$ & & & \\
\hline Coreopsis grandiflora & ${ }^{\star} 15$ & & & $x$ & & \\
\hline Coryzia bonariensis & 18 & $\mathrm{x}$ & $\mathrm{x}$ & & & \\
\hline Dahlia sp & 1415 & & $x$ & $x$ & & \\
\hline Emilia sonchifolia & 1115 & & $x$ & $x$ & $x$ & \\
\hline Eupatorium laevigatum & $* 15$ & & & $x$ & & \\
\hline E litorale & * 22 & & $x$ & & & \\
\hline Helianthus sp & $\star 15$ & & & $x$ & & \\
\hline Hypochoeris brasiliensis & 17 & & $x$ & & & \\
\hline Mikania sp & $\star 1$ & $\mathrm{x}$ & & ם & $x$ & \\
\hline M glomerata & ${ }^{*} 615$ & & & $\bar{x}$ & $\mathrm{x}$ & \\
\hline
\end{tabular}


Table II (continued)

\begin{tabular}{|c|c|c|c|c|c|c|}
\hline Bee plants & Ref & $\begin{array}{l}\text { Stingles } \\
\text { Meli- } \\
\text { pona }\end{array}$ & $\begin{array}{c}\text { s bees } \\
\text { Trigo } \\
\text { nini }\end{array}$ & $\begin{array}{c}\text { Africanized } \\
\text { Honeybees } \\
\text { A mellifera }\end{array}$ & $P$ & $N$ \\
\hline$M$ roraimensis & *2 2 & $x$ & & & & $x$ \\
\hline Senecio sp & 22 & & $x$ & & & \\
\hline$S$ brasiliensis & 15 & & & $x$ & & \\
\hline Soleosus & 22 & & $x$ & & & \\
\hline Sonchus sp & 15 & & & $x$ & & \\
\hline Soleraceus & 17 & & $x$ & & & \\
\hline Taraxacum officinale & +17 & $x$ & $x$ & & & \\
\hline Vernoniasp & ${ }^{*} 15$ & & & $x \boldsymbol{\square}$ & & \\
\hline$V$ canescens & $* 5$ & & $x$ & & & \\
\hline$\checkmark$ nitidula & $* 17$ & & $x$ & & & \\
\hline$V$ polyanthes & +15 & & & $x$ & & \\
\hline Vscabra & $* 1$ & $x$ & & & $x$ & \\
\hline Wedelia fertilis & 5 & & $x$ & & $x$ & $x$ \\
\hline $\begin{array}{l}\text { W paludosa } \\
\text { Convolvulaceae }\end{array}$ & 15 & & & $x$ & & \\
\hline $\begin{array}{l}\text { Convolvulaceae } \\
\text { lpomoea sp }\end{array}$ & ${ }^{\star} 4$ & & $x$ & & & \\
\hline $\begin{array}{l}\text { I cairica } \\
\text { Crassulaceae }\end{array}$ & $* 15$ & & & $x$ & & \\
\hline Bryophyllum sp & 11 & & $x$ & & $x$ & $x$ \\
\hline $\begin{array}{l}\text { Kalanchoe tubiflora } \\
\text { Cruciferae }\end{array}$ & 11 & & $x$ & & $x$ & \\
\hline $\begin{array}{l}\text { Raphanus sativus } \\
\text { Cucurbitaceae }\end{array}$ & 17 & $x$ & $x$ & & & \\
\hline $\begin{array}{l}\text { Momordica charantia } \\
\text { Cyperaceae }\end{array}$ & 723 & $x$ & $x$ & $x$ & $x$ & \\
\hline Cleome spinosa & 14 & $x$ & & & & \\
\hline Dilleniaceae & & & & & & \\
\hline $\begin{array}{l}\text { Davilla sp } \\
\text { Elaeocarnaceae }\end{array}$ & 3 & $x$ & $x$ & & $x$ & \\
\hline Muntigia calabura & 10 & & $x$ & & $x$ & \\
\hline Ericaceae & & & & & & \\
\hline Rhododendrum indicum & 1415 & $x$ & $x$ & & & \\
\hline R simsi & 4 & & $x$ & & & \\
\hline Euphorbiaceae & & & & & & \\
\hline Acalypha wilkesiana & 816 & $x$ & $x$ & & & $x$ \\
\hline Alchonea discolor & 23 & $x$ & & & $x$ & $x$ \\
\hline A latifolia & 5 & & $x$ & & & $x$ \\
\hline A sidaefolia & 910111316 & $\mathrm{x}$ & $x$ & & $x$ & $x$ \\
\hline A triplinervia & 12 & & $x$ & & & $x$ \\
\hline Croton sp & $* 22$ & & $x$ & & & \\
\hline Clundianus & * 1415 & $x$ & $x$ & $x$ & & \\
\hline C myrianthum & $* 4$ & & $x$ & & & \\
\hline Dalechampia sp & 1011 & & $x$ & & $x$ & \\
\hline Euphorbia milli & $* 1011$ & & $x$ & & $x$ & $x$ \\
\hline E prunifolia & $\star 17$ & $x$ & $x$ & & & \\
\hline Epulcherrima & ${ }^{*} 15$ & & & $x$ & $x$ & \\
\hline E splendens & $* 915$ & $x$ & $x$ & & $x$ & $x$ \\
\hline
\end{tabular}


Table II (continued)

\begin{tabular}{|c|c|c|c|c|c|c|}
\hline Bee plants & Ref & $\begin{array}{l}\text { Stingles } \\
\text { Meli- } \\
\text { pona }\end{array}$ & $\begin{array}{c}\text { Ss bees } \\
\text { Trigo- } \\
\text { nini }\end{array}$ & $\begin{array}{c}\text { Africanized } \\
\text { Honeybees } \\
\text { A mellifera }\end{array}$ & $P$ & $N$ \\
\hline Hura crepitans & 23 & & $x$ & $x$ & $x$ & \\
\hline Ricinus communis & 4611 & & $x$ & $x$ & $x$ & \\
\hline $\begin{array}{l}\text { Sebastiania cf serrata } \\
\text { Gramineae }\end{array}$ & 10 & & $x$ & & $x$ & \\
\hline $\begin{array}{l}\text { Coix lacrima-jobi } \\
\text { Guttiferae }\end{array}$ & 4 & & $x$ & & & \\
\hline Tovomitopsis nicaraguensis & 21 & $x$ & & & $x$ & \\
\hline $\begin{array}{l}\text { Vismia guianensis } \\
\text { Humiriaceae }\end{array}$ & 2 & $x$ & & & & $x$ \\
\hline $\begin{array}{l}\text { Humiria balsamifera } \\
\text { Iridaceae }\end{array}$ & 7 & $x$ & & & $x$ & \\
\hline Galidorea campestris & 4 & & $x$ & & & \\
\hline $\begin{array}{l}\text { Dietes vegeta } \\
\text { Labiatae }\end{array}$ & 15 & & & $x$ & & \\
\hline Coleus sp & 14 & $x$ & & & & \\
\hline Cumilla galioides & 22 & & $x$ & & & \\
\hline Hyptis brevipes & ${ }^{*} 2$ & $x$ & & & & $x$ \\
\hline Iboza riparia & 1415 & $x$ & $x$ & & & \\
\hline Leonorus sibiricus & +22 & & $x$ & & & \\
\hline Ocimum kilimandscharicum & 14 & $x$ & $x$ & $x$ & & \\
\hline Origanum vulgare & 15 & & & $x$ & & \\
\hline Salvia sp & ${ }^{*} 15$ & & $x$ & $\mathbf{\square}$ & & \\
\hline Sparanaensis & * 423 & & $x$ & & $x$ & \\
\hline $\begin{array}{l}\text { S splendens } \\
\text { Lecythidaceae }\end{array}$ & ${ }^{\star} 15$ & & $x$ & & & \\
\hline $\begin{array}{l}\text { Gustavia superba } \\
\text { Leguminosae }\end{array}$ & 20 & $x$ & & & $x$ & \\
\hline Acacia sp & * 101120 & $x$ & $x$ & $\mathbf{m}$ & $x$ & \\
\hline Acacia podalyriaefolia & $* 15$ & & & $\bar{x}$ & & \\
\hline Aeschynomene type & 6 & $x$ & & & $x$ & \\
\hline Andira anthelminthica & $* 11$ & & $x$ & $\mathrm{x}$ & $x$ & \\
\hline Bauhinia p & 15 & & & $x$ & & \\
\hline$B$ variegata & 15 & & $x$ & & & \\
\hline Cassia sp & ${ }^{*} 3$ & $x$ & $x$ & $\mathbf{\square}$ & $x$ & \\
\hline Cassia laevigata & * 422 & & $x$ & & & \\
\hline C siamea & +2 & $x$ & & & & $x$ \\
\hline Caesalpinia pelthophoroides & *612 & & $x$ & $x$ & $x$ & $x$ \\
\hline Calliandra brevipes & $\star 15$ & & & $x$ & & \\
\hline C tweedi & $* 15$ & & & $x$ & & \\
\hline Crotalaria lanceolata & 1415 & $x$ & $x$ & & & \\
\hline Dalbergia sp & $\star 12$ & & $x$ & & & $x$ \\
\hline Delonix regia & 23 & & $x$ & $x$ & $x$ & \\
\hline Desmodium sp & 15 & & & $x$ & & \\
\hline Erythrina speciosa & 15 & & & $x$ & & \\
\hline Inga edulis & * 115 & $x$ & & $x$ & $x$ & \\
\hline Leucaena glauca & 16 & & $x$ & & $x$ & \\
\hline L leucocephala & 6 & & & $x$ & $x$ & \\
\hline Lonchocarpus sp & 5 & & $x$ & & $x$ & $x$ \\
\hline
\end{tabular}


Table II (continued)

\begin{tabular}{|c|c|c|c|c|c|c|}
\hline \multirow[t]{2}{*}{ Bee plants } & \multirow[t]{2}{*}{ Ref } & \multicolumn{2}{|c|}{ Stingless bees } & \multirow{2}{*}{$\begin{array}{c}\text { Africanized } \\
\text { Honeybees } \\
\text { A mellifera }\end{array}$} & \multirow{2}{*}{$P$} & \multirow{2}{*}{$N$} \\
\hline & & $\begin{array}{l}\text { Mell- } \\
\text { pona }\end{array}$ & $\begin{array}{l}\text { Trigo- } \\
\text { nini }\end{array}$ & & & \\
\hline Mimosa sp & 7 & $x$ & & & $x$ & \\
\hline$M$ asperula type & *23 & $x$ & $x$ & & $x$ & \\
\hline$M$ bimucronata & $* 811$ & $x$ & & & & $x$ \\
\hline$M$ dalleoides & ${ }^{*} 13101115$ & $x$ & $x$ & $x$ & $x$ & $x$ \\
\hline$M$ dollens & $* 4$ & & $x$ & & & \\
\hline Minvisa & ${ }^{\star} 2$ & $x$ & & & & $\mathrm{x}$ \\
\hline$M$ micropteris & $* 4$ & & $x$ & & & \\
\hline$M$ aff pudica & $* 5$ & & $x$ & & & $x$ \\
\hline M pudica type & ${ }^{\star} 23$ & & & $x$ & $x$ & \\
\hline Mpudica & * 3723 & $x$ & & & $x$ & $x$ \\
\hline Mscabrella & +20 & $x$ & & $\mathbf{\square}$ & $x$ & \\
\hline$M$ velloziana & ${ }^{*} 681115$ & $x$ & & $x$ & $x$ & $x$ \\
\hline Myroxylum balsamum & 9111316 & $x$ & $\mathrm{x}$ & & $x$ & $\mathrm{x}$ \\
\hline Piptadenia sp & 91118 & $x$ & $x$ & $\mathbf{a}$ & $x$ & $x$ \\
\hline Pgonoacantha & 10161920 & $x$ & $x$ & $\mathbf{\square}$ & $x$ & $x$ \\
\hline$P$ rigida & 12 & & $x$ & & & $x$ \\
\hline Prosopis type & $* 23$ & $x$ & $x$ & & $x$ & \\
\hline Schizolobium sp & 20 & $x$ & & & $x$ & \\
\hline Sparahyba & 10 & $x$ & $x$ & & $x$ & \\
\hline Sesbania sp & 16 & & $x$ & & $x$ & \\
\hline Sophora tomentosa & 15 & & $x$ & & & \\
\hline Stryphnodendron guianensis & 3 & $x$ & $x$ & & $x$ & \\
\hline Tamarindus indica & +23 & & $x$ & & $x$ & \\
\hline Tipuana speciosa & +69101618 & $x$ & $x$ & $x$ & $x$ & \\
\hline Trifolium campestre & $* 17$ & & $x$ & & & \\
\hline Zornia sp & 15 & & & $x$ & & \\
\hline \multicolumn{7}{|l|}{ Liliaceae } \\
\hline Allium cepa & 17 & & $x$ & & & \\
\hline Aloe sp & ${ }^{*} 6910111516$ & & $x$ & & $x$ & \\
\hline Asparagus sl & 618 & & $x$ & & $\mathrm{x}$ & \\
\hline Asparagus-sp & 811 & $x$ & $x$ & & $x$ & $x$ \\
\hline A plumosus sl & 28 & $x$ & & & $x$ & $x$ \\
\hline $\begin{array}{l}\text { Hemerocallis fulva } \\
\text { Loranthaceae }\end{array}$ & 15 & & $x$ & & & \\
\hline Phthirusa micrantha & 2 & $x$ & & & & $x$ \\
\hline Lythraceae & & & & & & \\
\hline $\begin{array}{l}\text { Lafoensia nummularifolia } \\
\text { Malpighiaceae }\end{array}$ & 4 & & $x$ & & & \\
\hline Byrsonima sp & *2 & $x$ & & & $x$ & \\
\hline$B$ coriaceae & ${ }^{*} 7$ & $x$ & & & $x$ & \\
\hline$B$ intermedia & * 121516 & & $x$ & & $x$ & $x$ \\
\hline $\begin{array}{l}\text { Stigmaphyllon cf ellipticum } \\
\text { Malvaceae }\end{array}$ & 21 & & $\mathrm{x}$ & & $x$ & \\
\hline Sida sp & 5 & & $x$ & & $x$ & $x$ \\
\hline
\end{tabular}


Table II (continued)

\begin{tabular}{|c|c|c|c|c|c|c|}
\hline \multirow[t]{2}{*}{ Bee plants } & \multirow[t]{2}{*}{ Ref } & \multicolumn{2}{|c|}{ Stingless bees } & \multirow{2}{*}{$\begin{array}{c}\text { Africanized } \\
\text { Honeybees } \\
\text { A mellifera }\end{array}$} & \multirow[b]{2}{*}{$P$} & \multirow[b]{2}{*}{$N$} \\
\hline & & $\begin{array}{l}\text { Meli- } \\
\text { pona }\end{array}$ & $\begin{array}{c}\text { Trigo- } \\
\text { nini }\end{array}$ & & & \\
\hline \multicolumn{7}{|l|}{ Melastomataceae } \\
\hline Aciotis type & 23 & & & $x$ & $x$ & \\
\hline A dichotoma & 7 & $x$ & & & $x$ & \\
\hline Miconia sl & 8 & $x$ & & & $x$ & $x$ \\
\hline Miconia sp & 12311 & $x$ & $x$ & & $x$ & $x$ \\
\hline Miconia type & 13 & $x$ & & & $\mathrm{x}$ & $x$ \\
\hline$M$ cinerascens & 17 & $x$ & & & & \\
\hline Tibouchina type & 13 & $x$ & & & $x$ & $x$ \\
\hline Tibouchina sp & 68911 & $x$ & $x$ & $\boldsymbol{n}$ & $x$ & $x$ \\
\hline T chamissoana & 12 & & $x$ & & & $x$ \\
\hline Tholoserica & 15 & & $x$ & $x$ & & \\
\hline Tlongifolia & 5 & & $x$ & $\mathrm{x}$ & & \\
\hline \multicolumn{7}{|l|}{ Meliaceae } \\
\hline Cabralea sl & 18 & & $x$ & & & \\
\hline $\begin{array}{l}\text { Cedrela fissilis } \\
\text { Moraceae }\end{array}$ & 10 & & $x$ & & $x$ & \\
\hline Cecropia sp & 346918 & $\mathrm{x}$ & $x$ & $x \boldsymbol{x}$ & $x$ & $\mathrm{x}$ \\
\hline C adenopus & 1011 & $x$ & $x$ & $x$ & $x$ & $x$ \\
\hline C insignis & 21 & $x$ & & & $x$ & \\
\hline C obtusifolia & 5 & & $x$ & & $x$ & \\
\hline$C$ peltata & 23 & $\mathrm{x}$ & $x$ & $x$ & $x$ & \\
\hline Chlorophora tintoria & 23 & & $x$ & & $x$ & \\
\hline Coussapoa purpusii & 5 & & $x$ & & $x$ & \\
\hline Morus nigra & 91316 & $x$ & $x$ & & $x$ & \\
\hline Pourouma guianensis & 20 & & & $x$ & $x$ & \\
\hline $\begin{array}{l}\text { Sorocea bonplandii } \\
\text { Musaceae }\end{array}$ & 12 & & $x$ & & & $x$ \\
\hline Musa sp & +4 & & $x$ & & & \\
\hline \multicolumn{7}{|l|}{ Myrtaceae } \\
\hline Campomanesia guazumaefolia & 20 & $x$ & & E & $x$ & \\
\hline \multirow[t]{2}{*}{ Eucalyptus spp } & * 89101112 & & & & & \\
\hline & 13161819 & $x$ & $x$ & $x \square$ & $x$ & $x$ \\
\hline E blackelyi & ${ }^{*} 612$ & & $x$ & $x$ & $x$ & $x$ \\
\hline E cinerea & $* 12$ & & $x$ & & & $x$ \\
\hline E robusta & +612 & & $x$ & $x$ & $x$ & $x$ \\
\hline Erudis & ${ }^{*} 12$ & & $x$ & & & $x$ \\
\hline E tereticornis & +12 & & $x$ & & & $x$ \\
\hline Eugenia spp & $* 2320$ & $x$ & $\mathrm{x}$ & $\mathbf{\square}$ & $x$ & $x$ \\
\hline E pitanga & ${ }^{\star} 14$ & $x$ & & & & \\
\hline Myrciaria caulliflora & 19 & & $x$ & & $x$ & \\
\hline Psidium guajava sl & 8 & $x$ & & & & $x$ \\
\hline P guajava & +61013 & $x$ & & $x \mathbf{\square}$ & $\mathrm{x}$ & $x$ \\
\hline Syzygium cumini & +7 & $x$ & & & $x$ & \\
\hline S jambos & ${ }^{*} 15$ & & $x$ & & & \\
\hline \multicolumn{7}{|l|}{ Nyctaginaceae } \\
\hline Bouganvillea spectabilis & 8 & & $x$ & & & $\mathrm{x}$ \\
\hline Pisonia aculeata & 21 & & & $\mathrm{x}$ & $x$ & \\
\hline
\end{tabular}


Table II (continued)

\begin{tabular}{|c|c|c|c|c|c|c|}
\hline Bee plants & Ref & $\begin{array}{c}\text { Stingles } \\
\text { Meli- } \\
\text { pona }\end{array}$ & $\begin{array}{c}\text { Ss bees } \\
\text { Trigo- } \\
\text { nini }\end{array}$ & $\begin{array}{c}\text { Africanized } \\
\text { Honeybees } \\
\text { A mellifera }\end{array}$ & $P$ & $N$ \\
\hline \multicolumn{7}{|l|}{ Ochnaceae } \\
\hline $\begin{array}{l}\text { Ouratea sp } \\
\text { Oleaceae }\end{array}$ & 4 & & $x$ & & & \\
\hline Jasminum azoricum & 14 & $x$ & & & & \\
\hline \multicolumn{7}{|l|}{ Plamae } \\
\hline Archontophoenix sp & 1518 & & $x$ & $x$ & & \\
\hline A cunninghamia sl & 6 & & $x$ & & $x$ & \\
\hline A cunninghamia & 611 & & $x$ & & $x$ & $x$ \\
\hline Bactris gosipaes & 3 & & $x$ & & $x$ & \\
\hline Chamaedorea tepejilote & 5 & & $x$ & & $x$ & \\
\hline Cocos nucifera & +523 & $x$ & $x$ & $x$ & $x$ & $\mathrm{x}$ \\
\hline Elaeis guineensis & +5 & & $x$ & & $x$ & $x$ \\
\hline E oleifera & ${ }^{*} 20$ & $x$ & $x$ & $x$ & $x$ & \\
\hline Euterpe sp & 20 & $x$ & & & $x$ & \\
\hline Maximilliana martiana & 3 & $x$ & $x$ & & $x$ & \\
\hline $\begin{array}{l}\text { Socratea durissima } \\
\text { Phytollacaceae }\end{array}$ & 20 & $x$ & $x$ & $x$ & $x$ & \\
\hline Petiveria alliaceae & 5 & & $x$ & & $x$ & \\
\hline \multicolumn{7}{|l|}{ Polygonaceae } \\
\hline Polygonum acuminatum & ${ }^{*} 7$ & $x$ & & & $x$ & \\
\hline $\begin{array}{l}\text { Triplaris surinamensis } \\
\text { Proteaceae }\end{array}$ & +23 & $x$ & & & $x$ & \\
\hline Grevillea banksii & ${ }^{*} 15$ & & $x$ & $x$ & & \\
\hline Panopsis type & 7 & $x$ & & & & \\
\hline \multicolumn{7}{|l|}{ Rosaceae } \\
\hline Prunus sp & ${ }^{*} 22$ & & $x$ & & & \\
\hline P brasiliensis & $* 4$ & & $x$ & & & \\
\hline$P$ domestica & $* 17$ & & $x$ & & & \\
\hline$P$ persica & ${ }^{*} 15$ & & $x$ & & & \\
\hline Pyrus malus & 17 & & $x$ & & & \\
\hline Rubus urticaefolius & ${ }^{*} 17$ & & $x$ & & & \\
\hline \multicolumn{7}{|l|}{ Rubiaceae } \\
\hline Borreria laevis & ${ }^{\star} 2$ & $x$ & & & $x$ & \\
\hline B latifolia & ${ }^{*} 1 \overline{7}$ & & $x$ & & & \\
\hline Chimarris parviflora & 21 & $x$ & & & $x$ & \\
\hline Coffea arabica & +5 & & $x$ & $\mathbf{m}$ & & $x$ \\
\hline Diodia polymorpha & 15 & $x$ & & & & \\
\hline Plicourea sp & 22 & & $x$ & & & \\
\hline P marcgravii & 17 & & $x$ & & & \\
\hline Posoqueria latifolia & 20 & & $x$ & & $x$ & \\
\hline Warzewiczia coccinea & 2 & $x$ & & & $x$ & \\
\hline \multicolumn{7}{|l|}{ Rutaceae } \\
\hline Citrus sp & * 51015 & & $x$ & $x \boldsymbol{\square}$ & $x$ & $x$ \\
\hline
\end{tabular}


Table II (continued)

\begin{tabular}{|c|c|c|c|c|c|c|}
\hline Bee plants & Ref & $\begin{array}{c}\text { Stingles } \\
\text { Meli- } \\
\text { pona }\end{array}$ & $\begin{array}{c}\text { ss bees } \\
\text { Trigo- } \\
\text { nini }\end{array}$ & $\begin{array}{c}\text { Africanized } \\
\text { Honeybees } \\
\text { A mellifera }\end{array}$ & $P$ & $N$ \\
\hline \multicolumn{7}{|l|}{ Sapindaceae } \\
\hline Allophylus edulis & 17 & $x$ & & & & \\
\hline Sapindus saponaria & +5 & & $\mathrm{x}$ & & $x$ & \\
\hline $\begin{array}{l}\text { Serjania erecta } \\
\text { Sapotaceae }\end{array}$ & \multicolumn{6}{|c|}{ Sapotaceae } \\
\hline Pouteria stipitata & 21 & $x$ & & & $x$ & \\
\hline \multicolumn{7}{|l|}{ Saxifragaceae } \\
\hline Escallonia megapotamica & 22 & & $x$ & & & \\
\hline $\begin{array}{l}\text { Emontevidensis } \\
\text { Scrophulariaceae }\end{array}$ & 4 & & $x$ & & & \\
\hline Paulownia sp & 9 & & $\mathrm{x}$ & & $\mathrm{x}$ & \\
\hline P tomentosa & 12 & & $x$ & & $x$ & \\
\hline \multicolumn{7}{|l|}{ Solanaceae } \\
\hline Capsicum sp & 17 & $x$ & & & & \\
\hline Cestrum corymbosum & 422 & & $x$ & & & \\
\hline Cyphomandra sp & 17 & $\mathrm{x}$ & & & & \\
\hline Datura candida & 23 & & & $\mathbf{x}$ & $\mathrm{x}$ & \\
\hline Nicotiania tabacum & +15 & & $x$ & & & \\
\hline Solanum sp & 137810111720 & $x$ & $x$ & [ & $x$ & $x$ \\
\hline$S$ americanum & 17 & $x$ & & & & \\
\hline$S$ fiscifolium & 23 & & $x$ & $x$ & $x$ & \\
\hline$S$ sisymbriifolium & 17 & $x$ & $x$ & & & \\
\hline$S$ stramoniifolium & 7 & $x$ & & & $x$ & \\
\hline \multirow{2}{*}{\multicolumn{7}{|c|}{ Sterculiaceae }} \\
\hline & & & & & & \\
\hline Dombeya sp & $* 4$ & & $\mathrm{x}$ & & & \\
\hline$D$ burgessiae & * 1415 & $x$ & $x$ & $x$ & & \\
\hline D wallichi & * 1415 & $x$ & & $x$ & & \\
\hline Guazuma ulmifolia & 5 & & $x$ & & $\mathrm{x}$ & $x$ \\
\hline Sterculia chicha & 911 & & $x$ & & $x$ & \\
\hline \multicolumn{7}{|l|}{ Theaceae } \\
\hline Camellia japonica & 15 & & $x$ & & & \\
\hline \multicolumn{7}{|l|}{ Tiliaceae } \\
\hline \multicolumn{6}{|l|}{ Ulmaceae } & $x$ \\
\hline Trema micrantha & 3561012 & $x$ & $x$ & $x$ & $x$ & $x$ \\
\hline Ulmus mexicana & 5 & & $x$ & & $x$ & $x$ \\
\hline \multicolumn{7}{|l|}{ Umbelliferae } \\
\hline Centela biflora sl & 19 & & $x$ & & $x$ & \\
\hline Eryngium eburneum & 22 & & $\mathrm{x}$ & & & \\
\hline Foeniculum vulgare & 91115 & & $x$ & & $x$ & $x$ \\
\hline $\begin{array}{l}\text { Petroselium hortense } \\
\text { Velloziaceae }\end{array}$ & 12 & & $x$ & & $x$ & $x$ \\
\hline \multicolumn{6}{|l|}{ Verbenaceae } & \\
\hline Tectona grandis & 23 & & $x$ & & $\mathrm{x}$ & \\
\hline \multicolumn{7}{|l|}{ Violaceae } \\
\hline Rinorea squamata & 21 & $x$ & & & $x$ & \\
\hline \multicolumn{7}{|l|}{ Zingiberaceae } \\
\hline Alpinia henryi & 4 & & $x$ & & & \\
\hline
\end{tabular}


sources from the South of Brazil (South America) up to the South of Mexico (North America). In addition to the studies referred to in table I, some field studies (Sakagami et al, 1967; Sakagami and Laroca, 1971; Roubik, 1978; Laroca et al, 1982; Knoll, 1990), and an extensive survey of the principal pollen sources for European and Africanized honeybees in the Central and Western parts of Panama (Roubik et al, 1984) confirm this trend.

The families mentioned above comprise $\approx 60000$ species. Except for Compositae, Labiatae and Leguminosae, with 25000 , 3000 and 17000 species respectively, the rest are mainly distributed in tropical and subtropical regions all over the world (Joly, 1977; Heywood, 1978). Species of Compositae and Labiatae are very abundant in woodlands, wooded grasslands, grassland and bushland formations of South America, but are poorly represented in the tropical rain forests. Species of Caesalpinoideae and Mimosoideae (Leguminosae) are also more common in the tropics and subtropics. The neotropics are distinguished as a zone in which Solanaceae is concentrated, as the second largest concentration of Euphorbiaceae and Palmae, and the main center for dispersion of Myrtaceae, together with Australia, as well as being noted for the presence of Melastomataceae, one of the largest families in South America.

Thus when a general view is taken, it is evident that the main pollen and nectar sources for stingless bees are to be found among the plant families which are best represented in neotropical habitats. Regional differences can be seen, nonetheless. For example, the families Ericaceae, Labiatae and Polygonaceae are important for bees in the subtropics or in the zones where the tropics meet the subtropics, as shown by studies conducted in the South of Brazil (Sakagami and Laroca, 1971, La- roca et al, 1982, Orth, 1983; Knoll, 1990). Compositae and Labiatae predominate as bee plants in areas with open vegetation, often under anthropic influence. Palmae is more important in forested intertropical zones.

The following genera were important for both stingless bees and Africanized honeybees virtually over the entire neotropical zone: Alchornea, Baccharis, Cassia, Cecropia, Croton, Euphorbia, Miconia, Mimosa, Piptadenia, Solanum, Tibouchina, Tre$m a$ and Vernonia. The importance of Cecropia (Moraceae) is surprising in view of the supposedly anemophilous nature of this group, which is commonly found at the forest edge, on river banks, and in secondary forests throughout the neotropical zone. Piptadenia seems to be especially important for bees on the Atlantic coast of South America, from the South to the Northeast of Brazil. Other native genera frequently cited in the literature and which are regionally important are: Spondias (Anacardiaceae), Protium (Burseraceae), Euphorbia (Euphorbiaceae), Byrsonima (Malpighiaceae) and Eugenia (Myrtaceae).

Out of a total of approximately 288 plant species, 126, 52 and 25 plant species were important solely for Trigonini, Melipona and Africanized honeybee respectively. Fifty-three plant species were important both for Trigonini and Melipona. It should be noted that the information available for Melipona covers 13 species as opposed to that for Trigonini, which covers 36 species, so that the data are biased in favor of the latter group of bees. Finally, 58 species were simultaneously important for Africanized honeybees and stingless bees. In this case, there is an evident underestimation of the number of plant species that are important for both bee groups, in view of the small number of comparative studies (table 1). Be that as it may, when only comparative studies are considered, Trigonini may 
appear to be a more generalistic flower visitor than Melipona (Roubik, 1978; Orth, 1983; Knoll, 1990; and table II). Roubik (1978) also notes that Trigonini seem to be a more generalistic flower visitor than Africanized honeybees in forest and savanna habitats of Amazonia.

Preferential relationships of stingless bees with some plant families also seem possible (table II). If the frequency with which plant families are important in the various habitats or areas under study (table I) is considered, the following overall picture emerges: the families Leguminosae, Myrtaceae, Palmae and Rubiaceae are important for Africanized honeybees, Trigonini and Melipona; Anacardiaceae, Balsaminaceae, Compositae, Euphorbiaceae, Labiatae, Moraceae, Proteaceae and Sterculiaceae are more important for Africanized honeybees and Trigonini than for Melipona; Ericaceae, Liliaceae and Umbelliferae are more important for Trigonini than for Africanized honeybees and Melipona; Mimosoideae (Leguminosae), Melastomataceae, and Solanaceae are more important for Melipona than for Africanized honeybees and Trigonini. This kind of definition is obviously circumstantial and has to be experimentally verified.

Stingless bees have perennial colonies with specific populations of varying size but, with some exceptions (eg some species of Trigona), far smaller than those of Africanized honeybee colonies. Seen from a cost-benefit angle, for stingless bee colonies of a small size, it is an advantage to exploit flowers less frequently visited by those with a larger population, while for the latter it may well be that the adoption of strategies which give them more of a competitive edge in productive resources (Johnson and Hubbell, 1974, 1975; Hubbell and Johnson, 1978) is their main method for meeting their energy needs. Thus it is no surprise to find that in a given local context most of the food is obtained from only a few plant groups (table I), despite the huge number of important floral sources available on a regional scale and the vast plant diversity in tropical forest habitats (even when subject to anthropic influence).

As regards the impact of Africanized honeybees on neotropical bee plants, and particularly on stingless bees, there are forecasts of local extinction (Roubik, 1978; Roubik et al, 1986). Roubik (1989) admits that "Africanized honeybees seem preadapted to invade and persist in mosaic tropical habitats which gives them advantages over native bees", mostly owing to their generalized nesting habits. These bees are also better adapted than European honeybees to foraging in conditions of low nectar availability (Rinderer et al, $1984,1985)$, a situation which can be common at the forest edge and in open areas with unmanaged flora in the neotropics. Stingless bees are particularly abundant in regions of rain forest and subhumid forest zones of the neotropics, and are evidently adapted to the seasonal or apparently unforeseeable rhythms of flowering and variations in floral composition (as part of the high degree of diversity) in relatively close areas within neotropical forest habitats. It is not yet clear whether in such habitats the basis and pattern of food supply favors the Africanized honeybee's foraging strategies, although countless native plant species are important food sources for this bee.

\section{ACKNOWLEDGMENTS}

We wish to thank Mrs TC Giannini for help in elaborating the table. 
Résumé - Les plantes mellifères importantes pour les abeilles sans aiguillon (mélipones et trigones) et les abeilles africanisées dans les régions néotropicales : une synthèse. Cet article présente une synthèse des sources de nectar et de pollen pour les abeilles sans aiguilIon dans les régions néotropicales (Amérique du Sud, sauf Patagonie et Amérique centrale). Seules ont été prises en compte les informations quantitatives concernant les visites de fleurs, ce qui permet de comparer la valeur des sources florales spécifiques pour les abeilles sans aiguillon et les abeilles africanisées (tableau I). La plupart des données ont été obtenues par l'analyse pollinique de la nourriture récoltée par les abeilles. Quelques études de terrain ont été utilisées pour les listes de plantes visitées par les 2 groupes d'abeilles. Les études ont été faites dans des régions avec des types de végétation variés, influencés parfois par la présence d'agriculture, de prairies ou par l'urbanisation. Certaines ont été menées dans des forêts naturelles, très peu dans des régions d'herbages ou de savane.

Les familles botaniques suivantes : Anacardiacées, Composées, Euphorbiacées, Labiacées, Légumineuses, Mélastomatacées, Moracées, Myrtacées, Palmées, Rubiacées et Solanacées, qui renferment le plus grand nombre d'espèces importantes, à la fois pour les abeilles sans aiguillon et les abeilles africanisées (tableau II), sont aussi parmi celles qui présentent la plus grande diversité dans les habitats néotropicaux. Les genres Alchornea, Baccharis, Cassia, Cecropia, Croton, Euphorbia, Miconia, Mimosa, Piptadenia, Solanum, Tibouchina, Trema et Vernonia sont importants pour les 2 groupes d'abeilles dans pratiquement toute la zone néotropicale.

Sur un total de 288 espèces de plantes, 126,52 et 25 sont importantes pour uni- quement les trigones, les mélipones et les abeilles africanisées, respectivement. Cinquante trois espèces sont importantes pour les trigones et les mélipones à la fois. Les informations disponibles pour les mélipones couvrent 13 espèces, alors qu'elles en couvrent 36 pour les trigones; les données sont donc biaisées en faveur de ces dernières. Comme cela peut toujours se produire quand on ne fait que des études comparatives, les trigones semblent être plus généralistes que les mélipones dans leurs visites de fleurs. Cinquante huit espèces sont importantes pour les 2 groupes d'abeilles, abeilles sans aiguilion et abeilles africanisés. II y a une sousestimation évidente du nombre d'espèces importantes pour les 2 groupes, étant donné le faible nombre d'études comparatives (tableau I).

En ce qui concerne l'impact des abeilles africanisées sur les plantes mellifères néotropicales et en particulier sur les abeilles sans aiguillon, on peut prévoir l'extinction locale de plantes données. Mais l'on ne sait pas encore si la base et les modalités de l'offre de nourriture dans les habitats forestiers néotropicaux favorisent les stratégies de butinage des abeilles africanisées, bien que de nombreuses espèces de plantes indigènes soient d'importantes sources de nourriture pour ces abeilles.

abeille africanisée / Melipona / Trigonini/ plante mellifère / région néotropicale

Zusammenfassung - Wichtige Trachtptlanzen für Stachellose Bienen (Meliponen und Trigonen) und für Afrikanisierte Bienen an neotropischen Standorten : Eine Übersicht. Diese Arbeit bietet eine Übersicht über die Pollen- und Nektarquellen von Stachellosen Bienen an neotropischen (= südamerikanischen) 
Standorten. Es wurden nur zahlenmäßige Informationen über den Blütenbesuch berücksichtigt, wodurch ein Vergleich des Wertes spezifischer Trachtblüten für Stachellose Bienen und für Afrikanisierte Honigbienen möglich wird (Tabelle I). Die meisten Daten wurden durch die Pollenanalyse der von den Bienen gesammelten Nahrung gewonnen. Außerdem wurden einige Feldbeobachtungen zur Aufstellung von Listen der von beiden Bienengruppen besuchten Pflanzenarten herangezogen. Die Untersuchungen wurden in Gebieten mit unterschiedlicher Vegetation durchgeführt, in einigen Fällen beeinflußt durch landwirtschaftliche Nutzung, Viehweide oder städtische Siedlungen. Andere wurden in ursprünglichen Wäldern durchgeführt und sehr wenige in Gebieten mit Grasland oder Savanne.

Die Pflanzenfamilien Anacardiaceen, Compositen, Euphorbiaceen, Labiaten, Leguminosen, Melastomataceen, Moraceen, Myrtaceen, Palmae, Rubiaceen und Solanaceen enthalten einerseits die größte Zahl wichtiger Arten sowohl für die Stachellosen wie für die Afrikanisierten Bienen (Tabelle II), anderseits zeigen sie aber die größte Vielfalt an neotropischen Standorten. Die Gattungen Alchorea, Baccharis, Cassia, Cecropia, Croton, Euphorbia, Miconia, Mimosa, Piptadenia, Solanum, Tibouchina, Trema und Vernonia sind für beide Bienengruppen in praktisch der gesamten neotropischen Zone wichtig.

Aus einer Gesamtheit von 288 Pflanzenarten sind 126 ausschließlich für die Trigonen von Bedeutung, 52 nur für Meliponen und 25 nur für Honigbienen. 53 Arten sind sowohl für Trigonen wie für Meliponen wichtig. Die zur Verfügung stehenden Informationen über Meliponen beziehen sich auf 13 Arten, diejenigen über Trigonen jedoch auf 36 Arten, so daß diese Gruppe in unseren Daten stärker vertreten ist. Wie immer es sich verhalten mag, wenn man nur die Vergleichsstudien heranzieht, so ergibt sich, daß die Trigonen beim Blütenbesuch stärkere Generalisten sind als die Meliponen. Für beide Bienengruppen, Stachellose Bienen und Afrikanisierte Honigbienen, waren 58 Pflanzenarten gleichermaßen wichtige Trachtquellen. Es besteht eine deutliche Unterschätzung der Zahl von Trachtpflanzen, die für beide Gruppen von Bedeutung sind, besonders wenn man die geringe Zahl vergleichender Studien berücksichtigt (Tabelle I).

Was die Auswirkungen der Afrikanisierten Honigbienen auf neotropische Bienenpflanzen und besonders auf die heimischen Stachellosen Bienen betrifft, so gibt es Voraussagen für ein lokales Aussterben bestimmter Arten. Aber es ist noch keineswegs klar, ob die Basis und das Muster des Nahrungsangebots in neotropischen Waldstandorten die Sammelstrategie Afrikanisierter Bienen begünstigt. Es ist zu bedenken, daß dieser Biene zahllose heimische Pflanzenarten als wichtige Nahrungsquellen zur Verfügung stehen.

Afrikanisierte Biene / Melipona / Trigonini / Bienenpflanzen / neotropische Zone

\section{REFERENCES}

Absy ML, Kerr WE (1977) Algumas plantas visitadas para obtenção de pòlen por operàrias de Melipona seminigra merrillae em Manaus. Acta Amazônica 7, 309-315

Absy ML, Bezerra EB, Kerr WE (1980) Plantas meliferas utilizadas por 2 espècies de Melipona da Amazônia. Acta Amazônica 10, 271 281

Absy ML, Camargo JMF, Kerr WE, Miranda IP de A (1984) Espècies de plantas visitadas por Meliponinae (Hymenoptera; Apoidea) para coleta de pòlen na região do mèdio Amazonas. Rev Bras Biol 44, 227-237

Almeida MC de, Laroca S (1988) Trigona spinipes (Apidae, Meliponinae) : Taxonomia, Bi- 
onomia e relaçōes tròficas em àreas restritas. Acta Biol Par 17, 67-108

Arriaga ER (1989) Exploitacion de recursos florales por Plebeia sp (Apidae) en dos zonas con diferente altitud y vegetacion en el Soconusco, Chiapas. MSc Thesis, Faculdad de Ciencias, Universidad Nacional Autonoma de Mexico, Mexico

Barth OM (1970a) Anàlise microscòpica de algumas amostras de mel. I. Pòlen Dominante. An Acad Bras Ciênc 42, 351-366

Barth OM (1970b) Anàlise microscòpica de algumas amostras de mel. II. Pòlen acessòrio. An Acad Bras Ciênc 42, 571-590

Barth OM (1989) O Pôlen no mel Brasileiro. Rio de Janeiro, Gràfica Luxor, pp 150

Bortoli C de (1987) Estudo biocenòtico em Apoidea (Hymenoptera) de uma àrea restrita em São Josè dos Pinhais (Paranà, sul do Brasil), com notas comparativas. MSc Thesis, Departamento de Entomologia, Universidade Federal do Paranà, Curitiba, Brazil

Camargo JMF, Mazucato M (1984) Inventàrio da apifauna e flora apicola de Ribeirão Preto, SP, Brasil. Dusenia 14, 55-87

Camargo JMF, Moure JS (1988) Notas sobre Meliponinae (Hymenoptera, Apidae) colecionados por Felippo Silvestri na Bacia do Rio da Prata. Rev Bras Entomo/32, 293-324

Cortopassi-Laurino M (1982) Divisão de recursos tròficos entre abelhas sociais, principalmente em Apis mellifera Linnè e Trigona spiniped Fabricius (Apidae, Hymenoptera). PhD Thesis, Instituto de Biociências, Universidade de São Paulo, São Paulo, Brazil

Cortopassi-Laurino M, Ramalho M (1988) Pollen harvest by Africanized Apis mellifera and Trigona spinipes in Sao Paulo: botanical and ecological views. Apidologie 19, 1-24

Crane E, Walker P, Day R (1984) Directory of Important World Honey Sources. IBRA, London, pp 384

Engel MS, Dingemans-Bakels SF (1980) Nectar and pollen resources for stingless bees (Meliponinae, Hymenoptera) in Surinam (South America). Apidologie 11, 341-350

Guibu LS, Ramalho M, Kleinert-Giovannini A, Imperatriz-Fonseca VL (1988) Exploraçao dos recursos florais por colonias de Melipona quadrifasciata (Apidae, Meliponinae). Rev Bras Biol 48, 299-305
Hakim JRC (1983) Estudo ecològico da comunidade de abelhas silvestres (Hymenoptera, Apoidea) do Parque da Cidade, comparado com outras àreas de Curitiba, Paranà. MSc Thesis, Departamento de Entomologia, Universidade Federal do Paranà, Curitiba, Brazil

Heithaus ER (1979) Community structure of neotropical flower visiting bees and wasps: diversity and phenology. Ecology 60, 190-202

Heywood VH (1978) The Flowering Plants of the World. Oxford University Press, Oxford, pp 335

Hubbell SP, Johnson LK (1978) Comparative foraging behavior of six stingless bees species exploiting a standardized resource. Ecology 59, 1123-1136

Imperatriz-Fonseca VL (1989) Considerações sobre a ecologia das abelhas sociais. LivreDocente Thesis. Instituto de Biociências, Universidade de São Paulo, Brazil

Imperatriz-Fonseca VL, Kleinert-Giovannini $A$, Cortopassi-Laurino M, Ramalho M (1984) Hàbito de coleta de Tetragonisca angustula angustula Latreille (Apidae, Meliponinae). Bol Zool Univ, São Paulo 8, 115-131

Imperatriz-Fonseca VL, Kleinert-Giovannini $A$, Ramalho M (1987) Stingless bees and Africanized honeybees - resources sharing. In: Chemistry and Biology of Social Insects (Eder J, Rembold H, eds) Verlag J Peperny, Munich, pp 700-701

Imperatriz-Fonseca VL, Kleinert-Giovannini $A$, Ramalho M (1989) Pollen havest by eusocial bees in a non-natural community in Brazil. J Trop Ecol 5, 239-242

Iwama S, Melhem TS (1979) The pollen spectrum of the honey of Tetragonisca angustula angustula Latreille. Apidologie 10, 275-295

Joly AB (1977) Botànica - Introdução à Taxonomia Vegetal. Cia Editoria Nacional, Săo Paulo, pp 777

Johnson LK, Hubbell SP (1974) Aggression and competition among stingless bees: field studies. Ecology 55, 120-127

Johnson LK, Hubbell SP (1975) Contrasting foraging strategies and coexistence of two bee species on a single resource. Ecology 56, 1398-1406

Kleinert-Giovannini A, Imperatriz-Fonseca VL (1987) Aspects of the trophic niche of Melipo- 
na marginata marginata Lepeletier (Apidae, Meliponinae). Apidologie 18, 69-100

Kleinert-Giovannini A, Imperatriz-Fonseca VL, Ramalho M (1987) Exploitation of floral resources by Plebeia saiqui Friese (Apidae, Meliponinae). In : Chemistry and Biology of Social Insects (Eder J, Rembold H, eds) Verlag J Peperny, Munich, pp 157-159

Knoll FRN (1985) Abundància relativa das abelhas no campus da Universidade de São Pau-

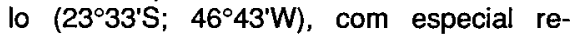
ferència à Tetragonisca angustula Latreille. MSc Thesis, Instituto de Biocièncias, Universidade de São Paulo, São Paulo, Brazil

Knoll FRN (1990) Abundància relativa, sazonalidade e preferèncias florais de Apidae (Hymenoptera), em uma ârea urbana $\left(23^{\circ} 33^{\prime} \mathrm{S}\right.$, $46^{\circ} 43^{\prime} \mathrm{W}$ ). PhD Thesis, Instituto de Biocièncias, Universidade de São Paulo, Brazil

Knoll FRN, Imperatriz-Fonseca VL (1987) Abundance and resources visited by individuals of Tetragonisca angustula. In : Chemistry and Biology of Social Insects (Eder J, Rembold $\mathrm{H}$, eds) Verlag J Peperny, Munich, pp 704705

Knoll FRN, Bego LR, Imperatriz-Fonseca VL (1987) Relative abundance and phenology of bees (Hymenoptera, Apoidea) in São Paulo, Brazil. In: Chemistry and Biology of Social Insects (Eder J, Rembold $\mathrm{H}$, eds) Verlag J Peperny, Munich, 702-703

Laroca S, Cure JR, Bortoli C de (1982) A associação de abelhas silvestres (Hymenoptera, Apoidea) de uma àrea restrita no interior da cidade de Curitiba (Brasil) : uma abordagem biocenòtica. Dusenia 13, 93-117

Louveaux J, Maurizio A, Vorwohl G (1978) Methods of melissopalynology. Bee World $59,139-157$

Mouga DMDS (1984) Atividade de coleta de Paratrigona subnuda (Moure) (Apidae, Meliponinae). MSc Thesis, Instituto de Biocièncias, Universidade de São Paulo, São Paulo, Brazil

Opler PA (1983) Nectar production in a tropical ecosystem. In: The Biology of Nectaries (Bentley B, Elias $T$, eds) Columbia Univ Press, N Y, pp 30-39

Orth Al (1983) Estudo ecològico de abelhas silvestres (Hymenoptera, Apoidea) em
Caçador, SC, com ènfase em polinizadores potenciais da macieira (Pyrus malus L) (Rosaceae). MSc Thesis, Departamento de Entomologia, Universidade Federal do Paranà, Curitiba, Brazil

Ramalho M (1987) Frequencia de uso de recursos florais por Scaptotrigona spp (Apidae, Meliponinae). MSc Thesis, Instituto de Biocièncias, Universidade de São Paulo, São Paulo, Brazil

Ramalho M (1990) Food harvesting by the stingless bee Scaptotrigona (Apidae, Meliponinae). J Apic Res (in press)

Ramalho $M$, Imperatriz-Fonseca VL, KleinertGiovannini A, Cortopassi-Laurino M (1985) Exploitation of floral resources by Plebeia remota (Holmberg) (Apidae, Meliponinae). Apidologie 16, 307-330

Ramalho M, Kleinert-Giovannini A, ImperatrizFonseca VL (1989) Utilization of floral resources by species of Melipona (Apidae, Meliponinae) : floral preferences. Apidologie 20, 185-195

Ramalho M, Guibu LS, Giannini TC, KleinertGiovannini A, Imperatriz-Fonseca VL (1990) Botanical origin of Brazilian honey. J Apic Res (submitted)

Rinderer TE, Bolten AB, Collins AM, Harbo JR (1984) Nectar-foraging characteristics of Africanized and European honey bees in the neotropics. J Apic Res 23, 70-79

Rinderer TE, Collins AM, Tucker KW (1985) Honey production and underlying nectar harvesting activities of Africanized and European honeybees. J Apic Res 27, 161-167

Roubik DW (1978) Competitive interactions between neotropical pollinators and Africanized honeybees. Science 201, 1030-1032

Roubik DW (1979) Africanized honeybees, stingless bees and the structure of tropical plant-pollinator communities. Proc IVth Symp on Pollination. Md Agric Exp Stn Spec Misc Publ 1, 403-417

Roubik DW (1989) Ecology and Natural History of Tropical Bees. Cambridge University Press, Cambridge. pp 514

Roubik DW, Schmalzel RJ, Moreno H (1984) Estudio Apibotanico de Panama: Cosecha y fuentes de pòlen y nectar usados por Apis mellifera $Y$ sus patrones estacionales $Y$ anu- 
ales. Organismo Internacional Regional de Sanidad Agropecuaria. Bol Tec 24

Roubik DW, Moreno JE, Vergara L, Wittmann D (1986) Sporadic food competition with the African honey bee: projected impact on neotropical social bees. $J$ Trop Ecol 2, 97-111

Sakagami SF, Laroca S (1971) Relative abundance phenology and flower visits of apid bees in eastern Parana, Southern Brazil. (Hymenoptera, Apoidea). Kontyo, 39, 217230

Sakagami SF, Laroca S, Moure JS (1967) Wild bee biocoenotics in São Josè dos Pinhais (PR), South Brasil. Preliminary report. J Fac Sci Hokkaido Univ Zool 16, 253-291
Santos CFO (1974) Anàlise polìnica de alguns mèis do estado de São Paulo. São Paulo, III Congresso Brasileiro de Apicultura, 273-278

Silveira FA, Garcia MVB, Moraes HC, Vieira MF, Campos LAO, llg $R$, Felipe MGA, Madsen VAH, Arzabe C (1984) Aspectos da competiçăo por fontes de pòlen entre Apis mellifera, Trigona spinipes e Partamona cupira (Hymenoptera : Apidae). Ciènc Cult 36 (suppl), 833

Sommeijer MJ, Rooy GA de, Punt W, Bruijn LLM de (1983) A comparative study of foraging behavior and pollen resources of various stingless bees (Hym, Meliponinae) and honeybees (Hym, Apinae) in Trinidad, West Indies. Apidologie 14, 205-224 